Proc. Indian Acad. Sci. (Chem. Sci.), Vol. 105, No. 6, December 1993, pp. 343-352.

(C) Printed in India.

\title{
Gold-platinum bimetallic cluster catalysts for visible light-induced hydrogen production from water
}

\author{
NAOKI TOSHIMA* and TETSU YONEZAWA \\ Department of Industrial Chemistry, Faculty of Engineering, The University of Tokyo, \\ Hongo, Bunkyo-ku, Tokyo 113, Japan
}

\begin{abstract}
Simultaneous reduction of two kinds of noble metal ions by refluxing in alcohol in the presence of poly(N-vinyl-2-pyrrolidone) can generally give polymer-protected bimetallic clusters with a core structure. The structures of the clusters were determined by the EXAFS measurements. On the other hand, it is known that micelle-protected platinum clusters work as good catalysts for visible light-induced hydrogen production from water

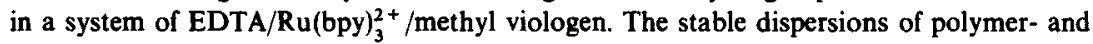
micelle-protected gold/platinum bimetallic clusters were prepared by alcohol- and photoreduction, respectively. The dispersions prepared by photoreduction in the presence of micelle are mainly composed of the mixtures of monometallic gold and platinum clusters, which cannot work as more active catalysts than monometallic platinum clusters. In contrast, the dispersions prepared by alcohol-reduction in the presence of polymer are composed of $\mathrm{Au} / \mathrm{Pt}$ bimetallic clusters with a "Pt-surrounded Au-core" structure, which are more active catalysts than the monometallic Pt clusters as catalysts for the hydrogen production.
\end{abstract}

Keywords. Bimetallic cluster; platinum; hydrogen production; catalysts; solar energy storage; electron transfer.

\section{Introduction}

Production of hydrogen and oxygen from water by using visible light energy has been a dream for mankind for artificial photosynthesis (see for example, Yamada et al 1983). Production of hydrogen by visible light irradiation in the system of

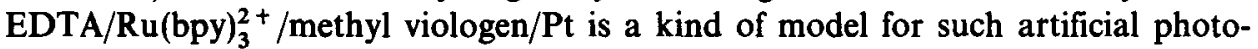
synthesis (figure 1). Although this system uses a sacrificial electron donor, EDTA, the production of hydrogen by visible light irradiation is an uphill reaction. In this system, platinum has been used as a catalyst for the production of hydrogen. Colloidal platinum is superior to other platinum catalysts like platinum black and conventional platinum catalysts because of its high specific surface area and transparency with respect to visible light.

More than a decade ago, we developed a convenient method to prepare colloidal dispersions of noble metals (Hirai et al 1978; Hirai and Toshima 1986). Refluxing of the solution of noble metal ions in alcohol without additives usually results in the formation of black precipitate which is the reduced metal. However, the addition of

* For correspondence 


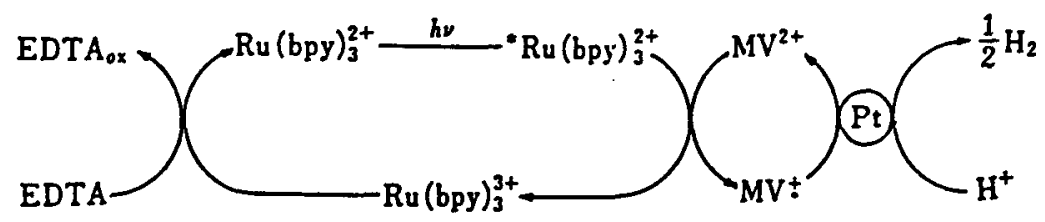

Figure 1. Visible light-induced hydrogen production in a system of Pt colloid/methyl viologen/ruthenium complex/EDTA (sacrificial reagent).

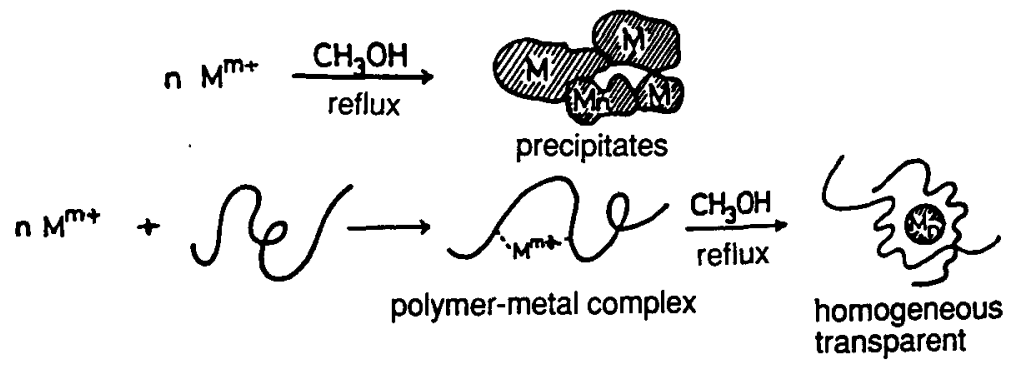

Figure 2. Schematic illustration of reduction of metal ions by refluxing in methanol in the absence and presence of poly( $\mathrm{N}$-vinyl-2-pyrrolidone).

a water-soluble polymer, especially poly( $\mathbf{N}$-vinyl-2-pyrrolidone), to the solution before reduction results in polymer-metal complexes which produce the homogeneous and transparent colloidal dispersion of noble metals on refluxing as illustrated in figure 2. Colloidal dispersions thus prepared contain metal clusters which are small in size and of narrow size distribution. They are stable and active as catalysts for months. The size of the clusters can be controlled by the reaction conditions, such as the type of alcohol or polymer used, and the concentration of the metal ions or alcohols. The colloidal dispersion of platinum clusters prepared by this method has been applied to the catalyst for the system shown in figure 1 , indicating an interesting size effect of the Pt-clusters upon the catalytic activity (Toshima et al 1981).

More sophisticated platinum catalysts have been developed by using surfactants (Toshima et al 1988). Thus, visible-light irradiation of the aqueous solution of hexachloroplatinic acid containing surfactant micelles gave a colloidal dispersion of platinum clusters protected by micelles. If the surfactant has a terminal olefin, UVirradiation in the presence of an initiator or $\gamma$-ray irradiation polymerizes the surfactant molecules to form polymerized micelle-protected platinum clusters. When the platinum clusters thus prepared are used as catalysts for visible light-induced hydrogen production in the system of figure 1 , the presence of the hydrophobic micellar phase in a hydrophilic aqueous medium can promote effective charge separation (figure 3).

Against this background, we would like to present here the preparation and characterization of bimetallic clusters, and the application of gold/platinum bimetallic clusters to the visible light-induced hydrogen production from water.

\section{Preparation and characterization of colloidal dispersions of bimetallic clusters}

Colloidal dispersions of bimetallic clusters can be easily prepared by refluxing the mixed solution of both metal ions in alcohol in the presence of poly(N-vinyl-2- 


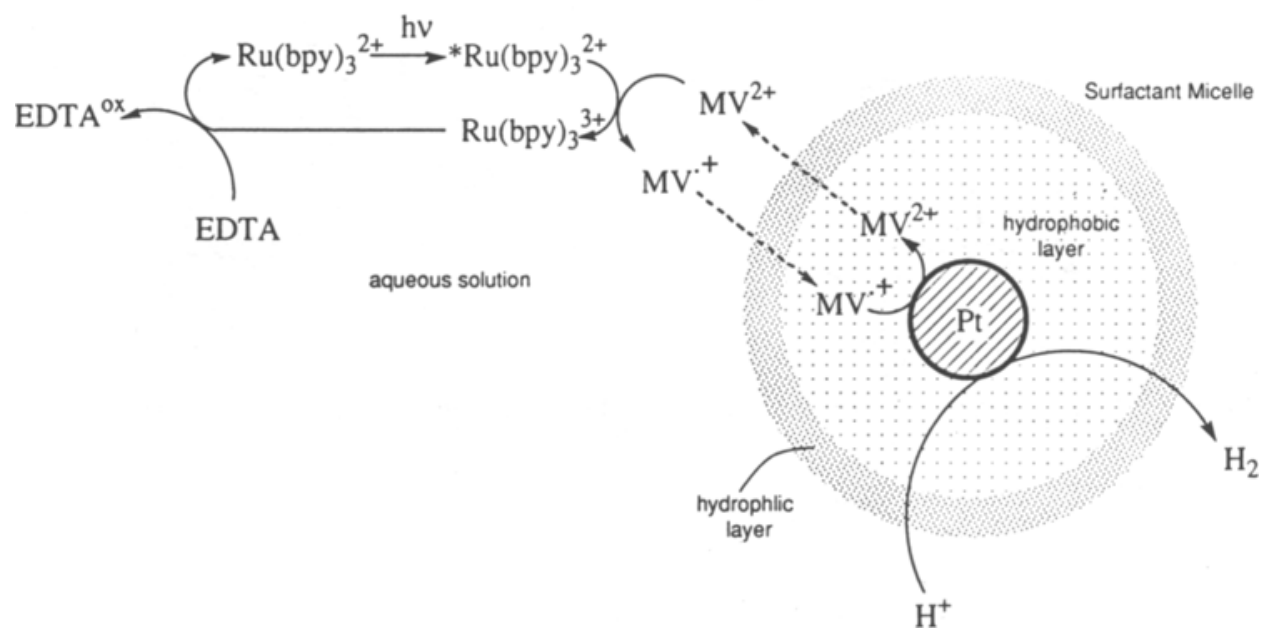

Figure 3. Schematic illustration of effective visible light-induced hydrogen production by using micelle-protected $\mathrm{Pt}$ clusters as catalyst.

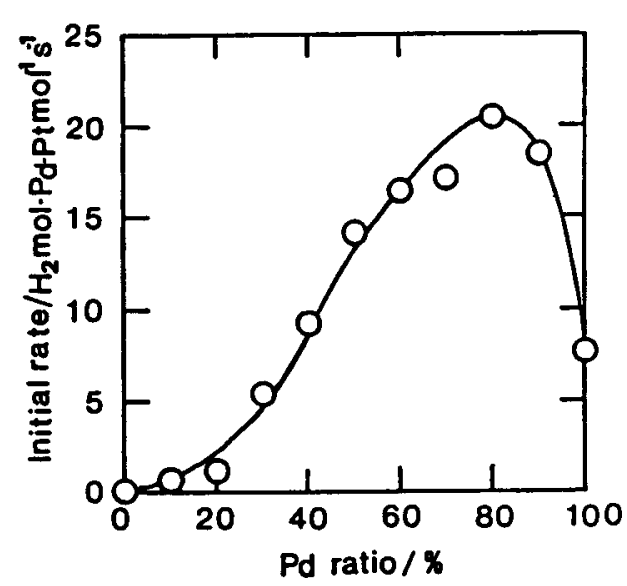

Figure 4. Dependence upon the metal composition of the catalytic activity of $\mathrm{Pd} / \mathrm{Pt}$ bimetallic clusters for partial hydrogenation of 1,3-cyclooctadiene.

pyrrolidone) (PVP). For example, the solution of $\mathrm{PdCl}_{2}$ and $\mathrm{H}_{2} \mathrm{PtCl}_{4}$ in ethanol/water $(1 / 1, \mathrm{v} / \mathrm{v})$ in the presence of the PVP was refluxed for $1 \mathrm{~h}$, resulting in colloidal dispersions of the $\mathrm{Pd} / \mathrm{Pt}$ bimetallic clusters (Toshima et al 1989). The bimetallic clusters are smaller in size and narrower in size distribution than monometallic platinum or palladium clusters. The $\mathrm{Pd} / \mathrm{Pt}$ bimetallic clusters thus prepared were used as catalysts for partial hydrogenation of 1,3-cyclooctadiene to cyclooctene. The dependence of catalytic activity on the metal composition (figure 4) indicates that the $\mathrm{Pd} / \mathrm{Pt}$ bimetallic cluster at a $\mathrm{Pd}$ concentration of $80 \%$, i.e., $\mathrm{Pd} / \mathrm{Pt}(4 / 1)$, has the highest activity, about 3 times that of the monometallic palladium cluster (Toshima et al 1989).

The structure of $\mathrm{Pd} / \mathrm{Pt}(4 / 1)$ bimetallic clusters has been determined by using an EXAFS technique (Toshima et al 1990, 1991; Harada et al 1992). Four kinds of coordination numbers obtained from EXAFS measurements for Pd/Pt (4/1) clusters 


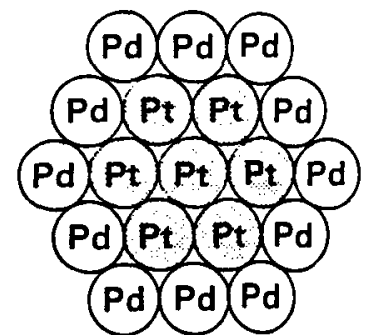

Figure 5. Model for Pd/Pt (4/1) bimetallic clusters.

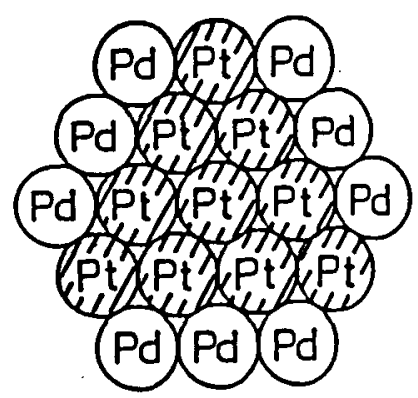

Figure 6. Model for Pd/Pt (1/1) bimetallic clusters.

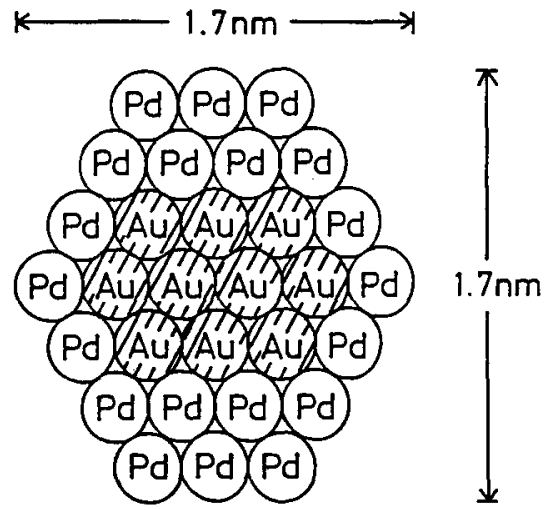

Figure 7. Model for Au/Pd (1/4) bimetallic clusters.

can be compared with those calculated for the model structure shown in figure 5 , indicating that the $\mathrm{Pd} / \mathrm{Pt}$ (4/1) cluster has a "Pd-surrounded Pt-core" structure. Even though the $\mathrm{Pd} / \mathrm{Pt}$ ratio is varied, the Pt-core structure is maintained. Thus, the $\mathrm{Pd} / \mathrm{Pt}(1 / 1)$ cluster has the structure shown in figure 6. In this structure, some of the $\mathrm{Pd}$ atoms located on the surface of the $\mathrm{Pd} / \mathrm{Pt}$ (4/1) cluster have been replaced by $\mathrm{Pt}$ atoms, but the other $\mathrm{Pd}$ atoms still remain on the surface forming islands.

$\mathrm{Au} / \mathrm{Pd}$ bimetallic clusters were also prepared by the same method as described above. Their structures were analyzed by the EXAFS technique again. Thus, the $\mathrm{Au} / \mathrm{Pd}(1 / 4)$ bimetallic cluster was shown to be a Pd-surrounded Au-core as shown in figure 7 (Toshima et al 1992). In a similar way, the $\mathrm{Rh} / \mathrm{Pt}$ (1/1) bimetallic cluster has been determined to be an assembly of microclusters with Rh-surrounded Pt-core structure.

From these results, it can be concluded that there is a tendency for noble metals to form the inner core thus: (inner) $\mathrm{Au}>\mathrm{Pt}>\mathrm{Pd}>\mathbf{R h}$ (outer). This tendency could be attributed to the coordination ability of the metal to the PVP which surrounds the metal cluster for protection.

\section{Colloidal dispersion of Au/Pt bimetallic clusters}

Colloidal dispersions of $\mathrm{Au} / \mathrm{Pt}$ bimetallic clusters were prepared by simultaneous photoreduction of $\mathrm{HAuCl}_{4}$ and $\mathrm{H}_{2} \mathrm{PtCl}_{6}$ in water in the presence of nonionic sur- 
factants $\mathrm{C}_{12} \mathrm{EO}$ and by simultaneous alcohol reduction of $\mathrm{HAuCl}_{4}$ and $\mathrm{H}_{2} \mathrm{PtCl}_{6}$ in ethanol/water in the presence of the water-soluble polymer, poly(N-vinyl-2-pyrrolidone) (PVP). The former method was used to obtain micelle-protected $\mathrm{Au} / \mathrm{Pt}$ bimetallic clusters and the latter, to obtain polymer-protected $\mathrm{Au} / \mathrm{Pt}$ bimetallic clusters. These clusters used for catalysis of visible light-induced hydrogen production from water in the system EDTA/Ru(bpy $)_{3}^{2+} /$ methyl viologen.

\subsection{Photoreduction system}

The degassed solution of $\mathrm{H}_{2} \mathrm{PtCl}_{6}$ and $\mathrm{HAuCl}_{4}$ in water in the presence of polyethylene glycol monolaurate $\left(C_{12} E O\right)$ was irradiated by visible light under argon for $4 \mathrm{~h}$, resulting in reddish-brown solution. The bimetallic dispersion samples thus obtained were used as the catalyst for visible light-induced hydrogen production. The results are shown in figure 8, along with those of physical mixtures of monometallic Au and Pt clusters prepared separately by the same method, and those of the monometallic $\mathrm{Pt}$ clusters at concentrations equal to the $\mathrm{Pt}$ fraction of the $\mathrm{Au} / \mathrm{Pt}$ bimetallic clusters. The comparison of these three kinds of catalysts indicates that the physical mixtures have the same activities as those of the monometallic Pt clusters, which means that the coexisting Au clusters have no effect on the catalytic activity of the monometallic $\mathrm{Pt}$ clusters. On the other hand, the Au/Pt bimetallic samples result in slightly higher catalytic activities than monometallic Pt clusters or mixtures. The increase in the activity is not as large as expected. This is actually because $\mathrm{Au} / \mathrm{Pt}$ bimetallic samples are mainly composed of monometallic Pt and monometallic Au clusters, and contain the $\mathrm{Au} / \mathrm{Pt}$ bimetallic clusters only in small fractions. This is confirmed by comparing the particle size distribution of the $\mathrm{Au} / \mathrm{Pt}(3 / 2)$ bimetallic sample with that of monometallic $\mathrm{Au}$ clusters. Thus, in the $\mathrm{Au} / \mathrm{Pt}$ bimetallic sample, the large particles are mainly composed of Au clusters and the small particles are the Pt clusters. Some particles in the intermediate size range are probably the $\mathrm{Au} / \mathrm{Pt}$ bimetallic clusters.

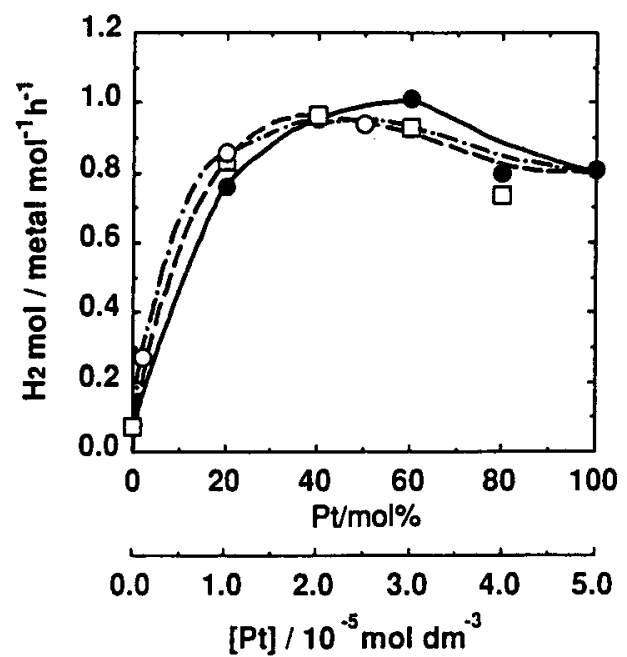

Figure 8. Visible light-induced hydrogen production catalyzed by $\mathrm{C}_{12}$ EO-protected $\mathrm{Au} / \mathrm{Pt}$ bimetallic clusters (O), physical mixtures of monometallic $A u$ and Pt clusters ( $\square$ ), and monometallic $\mathrm{Pt}$ clusters at concentrations equal to the $\mathrm{Pt}$ fraction of Au/Pt bimetallic clusters (O). 

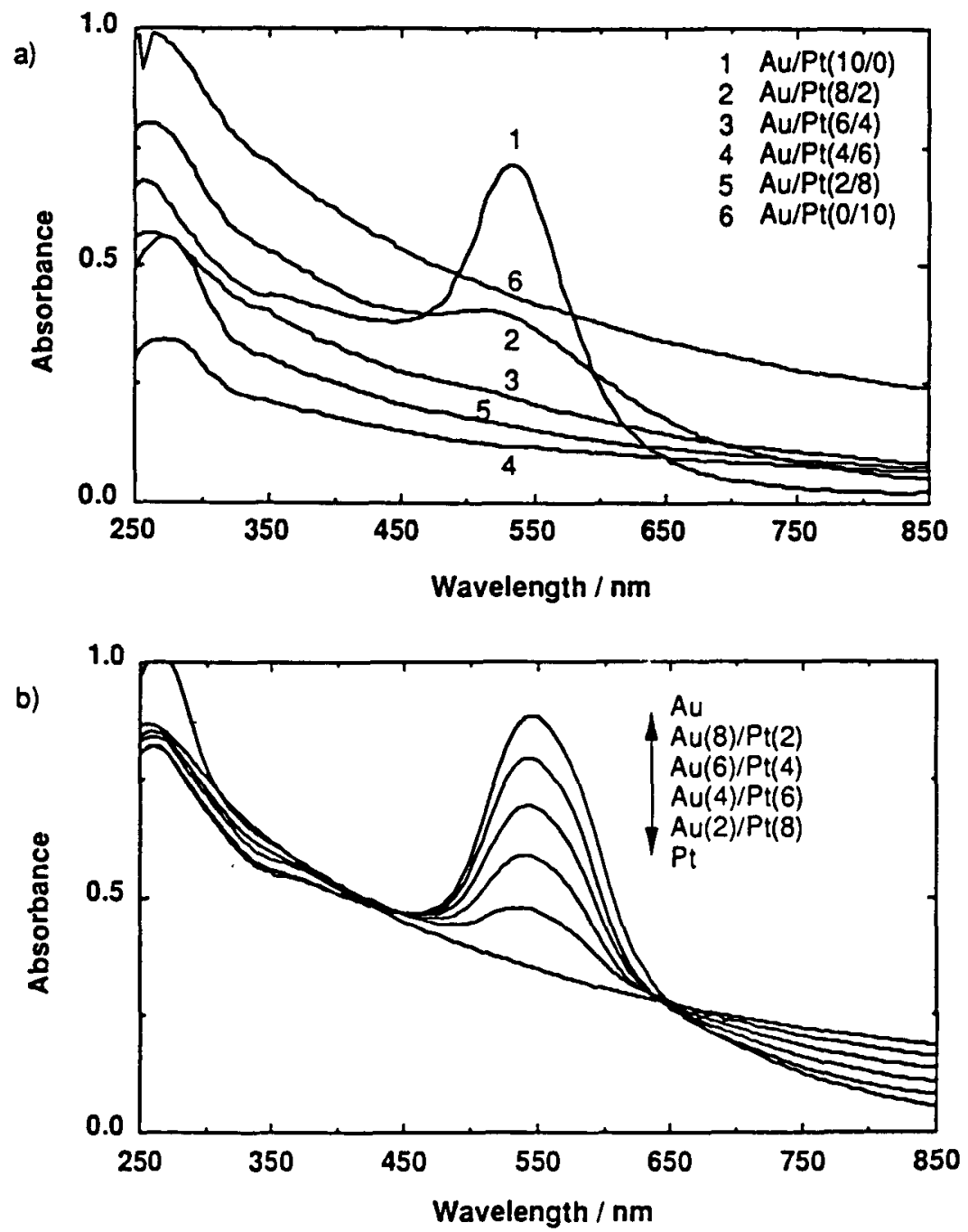

Figure 9. UV-Vis absorption spectra of (a) PVP-protected Au/Pt bimetallic clusters and (b) mixtures of $\mathrm{Au}$ and $\mathrm{Pt}$ monometallic clusters.

The same conclusion can be drawn by comparing the UV-Vis absorption spectra of the $\mathrm{Au} / \mathrm{Pt}$ bimetallic samples with those of the physical mixtures. The physical mixtures always have the absorption peak at about $545 \mathrm{~nm}$, which is attributed to the plasma absorption of $\mathrm{Au}$, the peak height of which corresponds to the fraction of Au clusters in the mixtures. The micelle-protected $\mathrm{Au} / \mathrm{Pt}$ bimetallic samples also have the same absorption peaks, although the peak height is not as high as that of the mixtures.

\subsection{Alcohol-reduction system}

The solution of $\mathrm{H}_{2} \mathrm{PtCl}_{6}$ and $\mathrm{HAuCl}_{4}$ in water/ethanol $(1 / 1, \mathrm{v} / \mathrm{v})$ in the presence of poly(N-vinyl-2-pyrrolidone) (PVP) was heated to refluxing under argon for $2 \mathrm{~h}$, 
resulting in a dark brownish solution (Toshima and Yonezawa 1992). The dispersions thus obtained are composed of small metal particles with rather narrow size distribution, as shown by observation with a transmission electron microscope (TEM).

The UV-Vis absorption spectra of a series of PVP-protected Au/Pt bimetallic clusters, shown in figure 9a, are quite different from those of the mixtures of monometallic $\mathrm{Au}$ and $\mathrm{Pt}$ clusters (figure $9 \mathrm{~b}$ ) and those of the $\mathrm{C}_{12}$ EO-protected $\mathrm{Au} / \mathrm{Pt}$
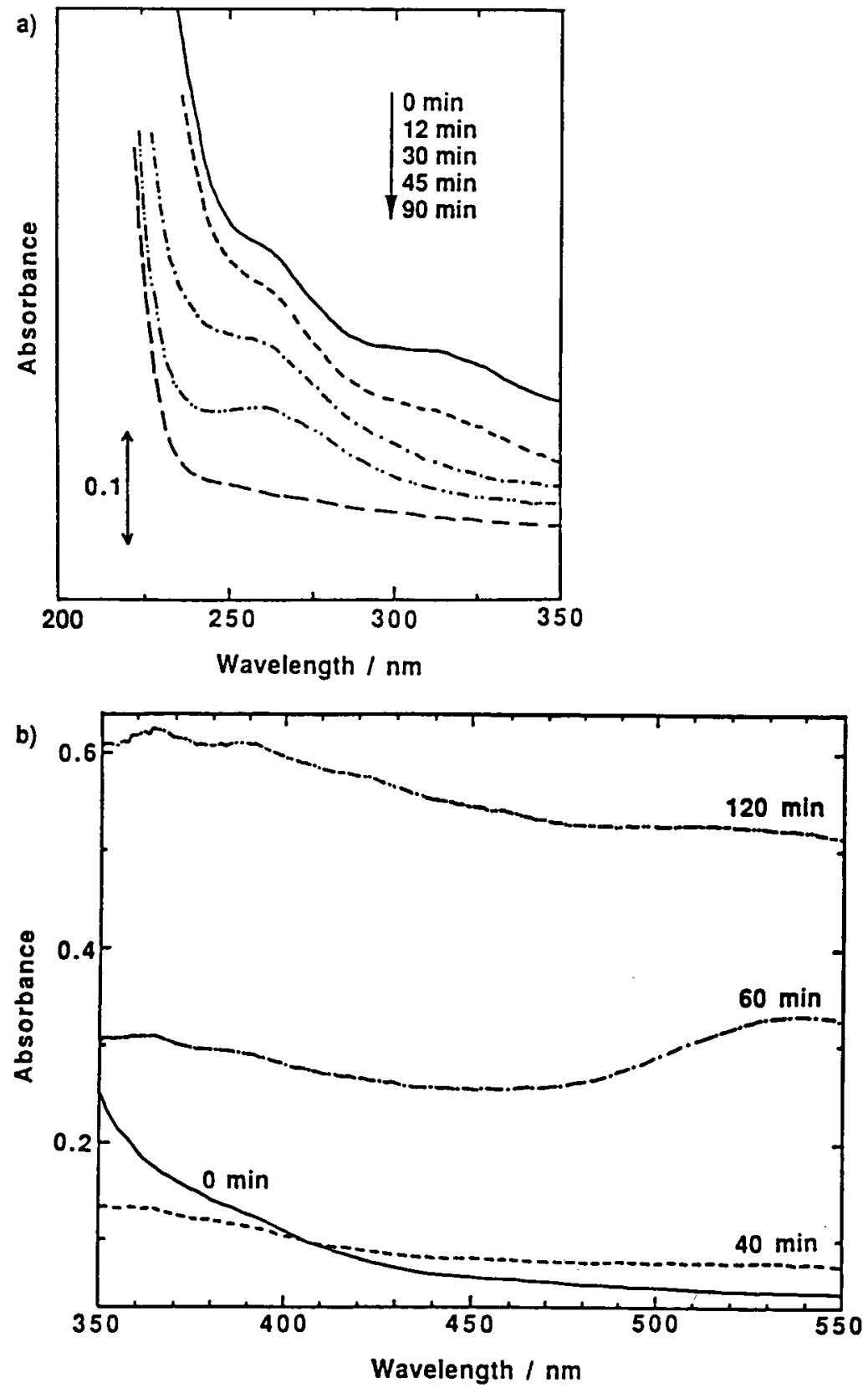

Figure 10. In situ UV-Vis absorption spectra during the reduction of a $1 / 1$ mixture of $\mathrm{HAuCl}_{4}$ and $\mathrm{H}_{2} \mathrm{PtCl}_{6}$ in ethanol/water (1/1) in the presence of PVP. $\lambda<350 \mathrm{~nm}$ (a) and $>350 \mathrm{~nm}$ (b). 

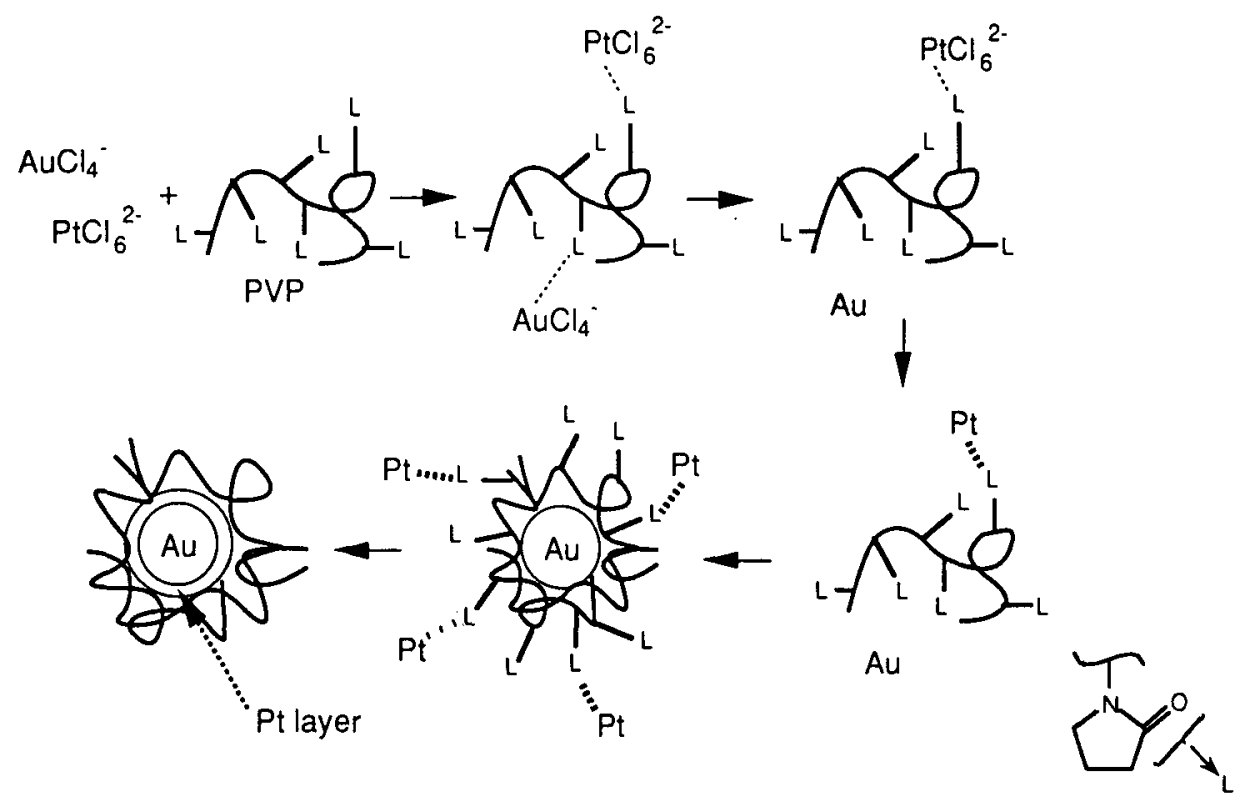

Figure 11. Schematic illustration of the formation by alcohol reduction of PVP-protected $\mathrm{Au} / \mathrm{Pt}$ bimetallic clusters.

clusters. Thus, the plasma absorption of Au decreases quickly with increase of $\mathrm{Pt}$ fraction in the $\mathrm{Au} / \mathrm{Pt}$ bimetallic clusters and completely disappears at $\mathrm{Au} / \mathrm{Pt}=3 / 2$. This demonstrates that enough $\mathrm{Au}$ atoms are not located on the surface of the $\mathrm{Au} / \mathrm{Pt}$ (3/2) bimetallic cluster particle to show plasma absorption. In other words, the $\mathrm{Au} / \mathrm{Pt}$ (3/2) cluster particle is mainly covered by $\mathrm{Pt}$ atoms, and the $\mathrm{Au}$ atoms are located near the centre of the bimetallic cluster particle, forming an Au core.

The Pt-surrounded Au-core structure is supported by the in situ UV-Vis absorption spectra during the reduction of a $1 / 1$ mixture of $\mathrm{HAuCl}_{4}$ and $\mathrm{H}_{2} \mathrm{PtCl}_{6}$ in the presence of PVP. As shown in figure 10, the spectrum changes with refluxing time. The change starts at the disappearance of the peak at about $310 \mathrm{~nm}$, due to $\mathrm{Au}$ (III) ions. Second, the peak at about $260 \mathrm{~nm}$, attributed to Pt(IV) ions, decreases, and third, the plasma absorption at $545 \mathrm{~nm}$ appears, indicating the formation of Au microclusters. At the last, however, plasma absorption disappears and the absorption at whole wavelength increases, suggesting the formation of Pt clusters. Thus, the Au microcluster is covered by $\mathrm{Pt}$ atoms forming particles with $\mathrm{Pt}$ atoms over the whole surface.

The process of formation of PVP-protected Au/Pt bimetallic clusters by alcohol reduction is schematically shown in figure 11 , by which the mechanism of formation of the Pt-surrounded Au-core structure can be understood. The importance of the coordination ability of PVP should be emphasized again in this case. The stronger coordination of PVP to Pt atoms as compared to Au atoms could control the order of the aggregation of the atoms, resulting in the Au core and then the $\mathrm{Pt}$ shell.

The PVP-protected Au/Pt bimetallic clusters were used as catalysts for visible light-induced hydrogen production. The results are shown in figure 12 for a total metal concentration of $7.5 \times 10^{-6} \mathrm{~mol} \mathrm{dm}^{-3}$. Comparison of the catalytic activity of the bimetallic clusters with that of monometallic $\mathrm{Pt}$ clusters indicates that $\mathrm{Au} / \mathrm{Pt}$ bimetallic clusters have higher activity than the monometallic $\mathrm{Pt}$ clusters. This is 


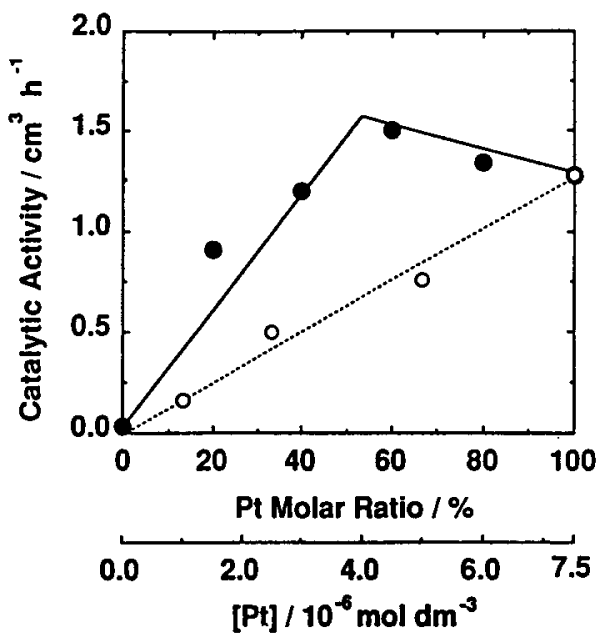

Figure 12. Catalytic activity depending on the metal composition and Pt concentration, respectively, of visible light-induced hydrogen production catalyzed by PVP-protected $\mathrm{Au} / \mathrm{Pt}$ bimetallic clusters ( $O$ ) and monometallic Pt clusters (O).

probably because of the ligand effect of the $\mathrm{Au}$ core on the $\mathrm{Pt}$ shell in the $\mathrm{Au} / \mathrm{Pt}$ bimetallic cluster particles. In other words, the Pt atoms on the surface of the bimetallic clusters could be rich in electron density by localization of electrons from the inner Au to the outer Pt because of the difference in electron affinities or ionization potentials of $\mathrm{Au}$ and Pt atoms.

\section{Conclusions}

(1) Alcohol reduction of mixtures of two kinds of noble metal ions in the presence of poly(N-vinyl-2-pyrrolidone) can produce poly( $\mathrm{N}$-vinyl-2-pyrrolidone)-protected bimetallic clusters with a core structure, although photoreduction of the same mixtures in the presence of micelles produces the complex mixtures.

(2) A common tendency has been observed for the metal which favors the inner core in the bimetallic clusters prepared by alcohol reduction in the presence of poly(Nvinyl-2-pyrrolidone): (inner) $\mathrm{Au}>\mathrm{Pt}>\mathrm{Pd}>\mathrm{Rh}$ (outer).

(3) Visible light-induced hydrogen production in a system of EDTA/Ru(bpy) $)_{3}^{+} /$ methyl viologen/metal catalyst cannot be accelerated by the coexistence of $\mathrm{Pt}$ and $\mathrm{Au}$ clusters, but can be accelerated by $\mathrm{Au} / \mathrm{Pt}$ bimetallic clusters with a "Pt-surrounded Au-core structure".

\section{Acknowledgements}

The authors express their sincere thanks to Dr M Harada, by whom part of the present work was done, and Drs $K$ Adachi and S Fukuda for their advice on TEM measurements. Financial support by a special grant from The Asahi Glass Foundation, and by Grants-in-Aid for Scientific Research in Priority Areas of "Macromolecular 
Complexes" and "Non-equilibrium Process in Solutions" from the Ministry of Education, Science and Culture, Japan are acknowledged.

\section{References}

Harada M, Asakura K, Ueki Y and Toshima N 1992 J. Phys. Chem. 969730

Hirai H, Nakao Y and Toshima N 1978 J. Macromol. Sci. Chem. A12 1117

Hirai H and Toshima N 1986 Tailored metal catalysts (ed.) Y Iwasawa (Dordrecht: Reidel) pp. 87-140

Toshima N, Harada M, Yamazaki Y and Asakura K 1992 J. Phys. Chem. 969927

Toshima N, Harada M, Yonezawa T, Kushihashi K and Asakura K 1991 J. Phys. Chem. 957448

Toshima N, Kuriyama M, Yamada Y and Hirai H 1981 Chem. Lett. 793

Toshima N, Kushihashi K, Yonezawa T and Hirai H 1989 Chem. Lett. 1769

Toshima N, Takahashi T and Hirai H 1988 J. Macromol. Sci. Chem. A25 669

Toshima N and Yonezawa T 1992 Makromol. Chem., Macromol. Symp. 59281

Toshima N, Yonezawa T, Harada M, Asakura K and Iwasawa Y 1990 Chem. Lett. 815

Yamada A, Toshima N and Kaneko M (eds) 1983 Photoenergy conversion (Tokyo: Sci. Soc. Press) 